\title{
NOVEL ROBUST STABILITY CRITERIA FOR UNCERTAIN STOCHASTIC NEURAL NETWORKS WITH TIME-VARYING DELAY
}

\author{
YONGGANG CHEN ${ }^{1}$, YUNRUI GUO ${ }^{1}$ AND WENLIN Li $^{2}$ \\ ${ }^{1}$ Department of Mathematics, Henan Institute of Science and Technology, \\ Xinxiang, Henan 453003, P.R. China \\ E-mail: happycygzmd@tom.com (Y.CHEN) \\ ${ }^{2}$ College of Mathematics and Information Science, Henan Normal University, \\ Xinxiang, Henan 453007, P.R. China \\ E-mail:liwenlin@henannu.edu.cn \\ Received: 01-02-2008 \\ Revised: 28-01-2009
}

\begin{abstract}
This paper considers the robust stability analysis problem for a class of uncertain stochastic neural networks with time-varying delay. Based on the Lyapunov functional method, and by resorting to the new technique for estimating the upper bound of the stochastic derivative of Lyapunov functionals, the novel asymptotic stability criteria are obatined in terms of Linear matrix inequalities (LMIs). Two numerical examples are presented to show the effectiveness and the less conservativeness of the proposed method.
\end{abstract}

Keywords: robust stability, stochastic neural networks, time-varying delay, linear matrix inequalities.

\section{Introduction}

The dynamics of neural networks has been extensively investigated in the past two decades due to their extensive applications in various signal processing problems such as optimization, fixed-point computations, and other areas. Among the most popular models in the literature of artificial neural networks is the continuous time model described by the following ordinary differential equations ${ }^{1}$

$$
\begin{array}{r}
C_{i} \frac{d u_{i}(t)}{d t}=-\frac{u_{i}(t)}{R_{i}}+\sum_{j=1}^{n} T_{i j} g_{j}\left(u_{j}(t)\right)+I_{i}, \\
i=1,2, \cdots, n,
\end{array}
$$

where $u_{i}(t)$ denotes the voltage on the input of neuron $i$ at time $t ; C_{i}>0$ and $R_{i}>0$ are the neuron amplifier input capacitance and resistance, respectively;
$I_{i}$ is the constant input from outside the system; matrix $T_{i j}$ is assumed to be irreducible, i.e. the network is strongly connected; function $g_{j}$ is the neuron activation function. This model was proposed by Hopfield with an electrical circuit implementation, and is referred to in the literature as a Hopfield-type neural network. In hardware implementation, time delay occurs due to the finite switching speeds of the amplifiers, which may induce the undesirable dynamic network behaviors such as oscillation, instability or other poor performances. Marcus and Westervelt ${ }^{2}$ introduced firstly a single time delay $\tau$ into (1). They considered the following system

$$
\begin{array}{r}
C_{i} \frac{d u_{i}(t)}{d t}=-\frac{u_{i}(t)}{R_{i}}+\sum_{j=1}^{n} T_{i j} g_{j}\left(u_{j}(t-\tau)\right)+I_{i} \\
i=1,2, \cdots, n .
\end{array}
$$


System (2) can be extended to the following models

$$
\begin{array}{r}
\frac{d u_{i}(t)}{d t}=-a_{i} u_{i}(t)+\sum_{j=1}^{n} b_{i j} g_{j}\left(u_{j}(t-\tau(t))\right)+I_{i}, \\
i=1,2, \cdots, n,
\end{array}
$$

and

$$
\begin{array}{r}
\frac{d u_{i}(t)}{d t}=-c_{i} u_{i}(t)+\sum_{j=1}^{n} a_{i j} g_{j}\left(u_{j}(t)\right) \\
+\sum_{j=1}^{n} b_{i j} g_{j}\left(u_{j}(t-\tau(t))\right)+I_{i}, \\
i=1,2, \cdots, n,
\end{array}
$$

where $\tau(t)$ denotes constant or time-varying delay. The stability analysis problem for delayed neural networks (3) and (4) has received great attention during the past years ${ }^{3-14}$. These obtained results can be classified into two types: delay-independent stability criteria and delay-dependent stability criteria. The former do not make use of information on the size of delay while the latter include such information. It is known that delay-dependent stability conditions are generally less conservative than delayindependent ones especially when the size of the delay is small.

On the other hand, in real nervous systems, the synaptic transmission is a noisy process brought on by random fluctuations from the release of neurotransmitters and other probabilistic causes, and it has been realized that a neural network could be stabilized or destabilized by certain stochastic inputs ${ }^{15}$ which leads to the research on dynamics of stochastic neural networks ${ }^{16-23}$. The robust stability criteria were established in Refs.18 and 19 for uncertain stochastic Hopfield neural networks with constant and time-varying delays. In Ref.23, the delaydependent robust stability analysis problem was investigated for uncertain stochastic neural networks with time-varying delay. The restrictions that the time-varying delay was differentiable and its derivative was less than one are removed in Ref. 23. However, the useful terms are ignored and the enlargement method is rather conservative in Ref. 23 when estimating the upper bound of the stochastic derivative of Lyapunov functionals.
In this paper, the delay-dependent robust stability problem is investigated for a class of uncertain stochastic neural networks with time-varying delay. Based on the Lyapunov functional method, and by reserving the useful terms when estimating the upper bound of the derivative of Lyapunov functionals, the novel stability criteria are established in terms of LMIs. Finally, two numerical example are presented to show that our results are less conservative than some existing ones.

Notation. The superscript " $T$ " stands for the transpose of a matrix. $\mathrm{R}^{n}$ and $\mathrm{R}^{n \times n}$ denote the $\mathrm{n}$ dimensional Euclidean space and set of all $n \times n$ real matrices, respectively. A real symmetric matrix $X>0(\geqslant 0)$ denotes $X$ being a positive definite (positive semi-definite) matrix. $(\Omega, \mathscr{F}, P)$ denotes a complete probability space, where $\Omega$ is a sample space, $\mathscr{F}$ is the $\sigma$-algebra subsets of the sample space and $P$ is the probability measure on $\mathscr{F}$. Denote by $L_{\mathscr{F}_{0}}^{2}\left([-\bar{\tau}, 0] ; \mathrm{R}^{n}\right)$ the family of all $\mathscr{F}_{0}$ measurable $C\left([-\bar{\tau}, 0] ; \mathrm{R}^{n}\right)$-valued random variables $\phi=$ $\{\phi(t):-\bar{\tau} \leqslant t \leqslant 0\}$ such that $\sup _{-\bar{\tau} \leqslant t \leqslant 0} \mathrm{E}|\phi(t)|^{2}<$ $\infty$, where $\mathrm{E}\{$.$\} stands for the mathematical expecta-$ tion operator with respect to $P$. $I$ is used to denote an identity matrix with proper dimension. Matrices, if not explicitly stated, are assumed to have compatible dimensions. The symmetric terms in a symmetric matrix are denoted by $*$.

\section{Problem formulation}

Consider the following uncertain stochastic neural network with time-varying delay described by

$$
\begin{aligned}
\mathrm{d} x(t)= & {\left[-(A+\Delta A) x(t)+\left(W_{0}+\Delta W_{0}\right) f(x(t))\right.} \\
& \left.+\left(W_{1}+\Delta W_{1}\right) f(x(t-\tau(t)))\right] \mathrm{d} t \\
& +[(C+\Delta C) x(t)+(D+\Delta D) \\
& \times x(t-\tau(t))] \mathrm{d} \omega(t),
\end{aligned}
$$

where $x(t)=\left[x_{1}(t), x_{2}(t), \cdots, x_{n}(t)\right]^{T} \in \mathrm{R}^{n}$ is the neuron state vector, and $\tau(t)$ is the time-varying delay which satisfies $0 \leqslant \tau(t) \leqslant \bar{\tau}, \dot{\tau}(t) \leqslant \mu . f(x(\cdot))=$ $\left[f_{1}\left(x_{1}(\cdot)\right) f_{2}\left(x_{2}(\cdot)\right), \cdots, f_{n}\left(x_{n}(\cdot)\right)\right]^{T} \in \mathrm{R}^{n}$ is the activation function, $\omega(t)=\left[\omega_{1}(t), \omega_{2}(t), \cdots, \omega_{m}(t)\right]^{T} \in$ $\mathrm{R}^{m}$ is a m-dimensional Brownian motion defined 
on a complete probability space $(\Omega, \mathscr{F}, P) . \quad A=$ $\operatorname{diag}\left\{a_{1}, a_{2}, \cdots, a_{n}\right\}$ is a diagonal matrix with $a_{i}>0$, and $W_{0}, W_{1}$ are the connection weight matrix and the delayed weight matrix, respectively. $C$ and $D$ are known real constant matrices. $\Delta A, \Delta W_{0}, \Delta W_{1}, \Delta C$ and $\Delta D$ denote the time-varying parameter uncertainties and are of the following form

$$
\begin{aligned}
& {\left[\begin{array}{lllll}
\Delta A & \Delta W_{0} & \Delta W_{1} & \Delta C & \Delta D
\end{array}\right]} \\
& =H F(t)\left[\begin{array}{lllll}
E_{1} & E_{2} & E_{3} & E_{4} & E_{5}
\end{array}\right],
\end{aligned}
$$

where $H, E_{1}, E_{2}, E_{3}, E_{4}, E_{5}$ are known real constant matrices with appropriate dimensions, and $F(t)$ is unknown time-varying matrix satisfying $F^{T}(t) F(t) \leqslant I$.

Throughout the paper, we make the following assumptions on the activation function in the delayed stochastic neural networks (5).

Assumption 2.1 Activation function $f():. \mathrm{R}^{n} \rightarrow \mathrm{R}^{n}$ is bounded and satisfies

$$
\|f(x)\| \leqslant\|G x(t)\|,
$$

for any $x(t) \in \mathrm{R}$, where $G \in \mathrm{R}^{n \times n}$ is a known matrix.

Assumption 2.2 Each activation function $f_{i}():. \mathrm{R} \rightarrow$ $\mathrm{R}$ is bounded and satisfies

$$
0 \leqslant \frac{f_{i}\left(\zeta_{1}\right)-f_{i}\left(\zeta_{2}\right)}{\zeta_{1}-\zeta_{2}} \leqslant k_{i}, \quad i=1,2, \cdots, n,
$$

for any $\zeta_{1}, \zeta_{2} \in \mathrm{R}, \zeta_{1} \neq \zeta_{2}$, where $k_{i}>0$ for $i=$ $1,2, \cdots, n$.

Let $x(t ; \phi)$ denote the state trajectory of stochastic neural network (1) from the initial function $x(t)=$ $\phi(t)$ on $-\bar{\tau} \leqslant t \leqslant 0$ in $L_{\mathscr{F}_{0}}^{2}\left([-\bar{\tau}, 0] ; \mathrm{R}^{n}\right)$. Under the assumption 2.1, it follows from Ref. 25 that the system (5) admits a trivial solution $x(t ; 0)=0$.

Before giving the main results, we will firstly introduce the following definition and lemmas.

Definition 2.3 The trivial solution of the stochastic neural network (5) is said to be globally robustly asymptotically stable in the mean square, if for all admissible uncertainties satisfying (6), the following equation holds

$$
\lim _{t \rightarrow \infty} \mathrm{E}|x(t ; \phi)|^{2}=0 .
$$

Lemma 2.4 For any real vectors $a, b$ and any matrix $Q>0$ with appropriate dimensions, it follows that

$$
2 a^{T} b \leqslant a^{T} Q a+b^{T} Q^{-1} b .
$$

Lemma $2.5^{19}$ For given matrices $H, E$ and $F$ with $F^{T} F \leqslant I$ and scalar $\varepsilon>0$, the following inequality holds

$$
H F E+E^{T} F^{T} H^{T} \leqslant \varepsilon^{-1} H H^{T}+\varepsilon E^{T} E .
$$

\section{Main results}

In this section, we will firstly present the global asymptotic stability criterion of the following nominal system without uncertainties, this is

$$
\begin{aligned}
\mathrm{d} x(t)= & {\left[-A x(t)+W_{0} f(x(t))+W_{1} f(x(t-\tau(t)))\right] \mathrm{d} t } \\
& +[C x(t)+D x(t-\tau(t))] \mathrm{d} \omega(t) .
\end{aligned}
$$

Theorem 3.1 For given constants $\bar{\tau} \geqslant 0, \mu \geqslant 0$. Under Assumption 2.1, the system (9) is globally asymptotically stable in the mean square, if there exist matrices $P>0, Q_{1}>0, Q_{2}>0, Q_{3}>0, R>0, S>$ $0, M_{i}, N_{i},(i=1,2, \cdots, 5)$, and scalars $\alpha>0, \beta>0$, such that the following linear matrix inequalities (LMIs) hold

$$
\begin{aligned}
& \Gamma_{1}<0, \\
& \Gamma_{2}<0,
\end{aligned}
$$

where

$$
\begin{aligned}
\Gamma_{1} & =\left[\begin{array}{ccccccc}
\Omega & \bar{\tau} \Xi_{1}^{T} R & \Xi_{2}^{T} P & \bar{\tau} \Xi_{2}^{T} S & M^{T} & N^{T} & \bar{\tau} M^{T} \\
* & -\bar{\tau} R & 0 & 0 & 0 & 0 & 0 \\
* & * & -P & 0 & 0 & 0 & 0 \\
* & * & * & -\bar{\tau} S & 0 & 0 & 0 \\
* & * & * & * & -S & 0 & 0 \\
* & * & * & * & * & -S & 0 \\
* & * & * & * & * & * & -\bar{\tau} R
\end{array}\right], \\
\Gamma_{2} & =\left[\begin{array}{ccccccc}
\Omega & \bar{\tau} \Xi_{1}^{T} R & \Xi_{2}^{T} P & \bar{\tau} \Xi_{2}^{T} S & M^{T} & N^{T} & \bar{\tau} N^{T} \\
* & -\bar{\tau} R & 0 & 0 & 0 & 0 & 0 \\
* & * & -P & 0 & 0 & 0 & 0 \\
* & * & * & -\bar{\tau} S & 0 & 0 & 0 \\
* & * & * & * & -S & 0 & 0 \\
* & * & * & * & * & -S & 0 \\
* & * & * & * & * & * & -\bar{\tau} R
\end{array}\right],
\end{aligned}
$$




$$
\begin{aligned}
\Omega & =\left[\begin{array}{ccccc}
\Omega_{11} & \Omega_{12} & \Omega_{13} & \Omega_{14} & \Omega_{15} \\
* & \Omega_{22} & \Omega_{23} & \Omega_{24} & \Omega_{25} \\
* & * & \Omega_{33} & -N_{4}^{T} & -N_{5}^{T} \\
* & * & * & \Omega_{44} & \Omega_{45} \\
* & * & * & * & \Omega_{55}
\end{array}\right], \\
\Xi_{1} & =\left[\begin{array}{lllll}
-A & 0 & 0 & W_{0} & W_{1}
\end{array}\right], \\
\Xi_{2} & =\left[\begin{array}{lllll}
C & D & 0 & 0 & 0
\end{array}\right], \\
M & =\left[\begin{array}{lllll}
M_{1}^{T} & M_{2}^{T} & M_{3}^{T} & M_{4}^{T} & M_{5}^{T}
\end{array}\right], \\
N & =\left[\begin{array}{lllll}
N_{1}^{T} & N_{2}^{T} & N_{3}^{T} & N_{4}^{T} & N_{5}^{T}
\end{array}\right],
\end{aligned}
$$

and

$$
\begin{aligned}
\Omega_{11} & =-P A-A^{T} P^{T}+Q_{1}+Q_{2}+M_{1} \\
& +M_{1}^{T}+\alpha G^{T} G, \\
\Omega_{12}= & -M_{1}+M_{2}^{T}+N_{1}, \\
\Omega_{13}= & M_{3}^{T}-N_{1}, \\
\Omega_{14} & =P W_{0}+M_{4}^{T}, \\
\Omega_{15} & =P W_{1}+M_{5}^{T}, \\
\Omega_{22} & =-(1-\mu) Q_{1}-M_{2}-M_{2}^{T}+N_{2} \\
& +N_{2}^{T}+\beta G^{T} G, \\
\Omega_{23} & =-M_{3}^{T}-N_{2}+N_{3}^{T}, \\
\Omega_{24} & =-M_{4}^{T}+N_{4}^{T}, \\
\Omega_{25} & =-M_{5}^{T}+N_{5}^{T}, \\
\Omega_{33} & =-N_{3}-N_{3}^{T}-Q_{2}, \\
\Omega_{44} & =-\alpha I+Q_{3}, \\
\Omega_{55} & =-\beta I-(1-\mu) Q_{3} .
\end{aligned}
$$

Proof. Set $y(t)=-A x(t)+W_{0} f(x(t))+W_{1} f(x(t-$ $\tau(t)))$ and $g(t)=C x(t)+D x(t-\tau(t))$, then system (9) becomes

$$
\mathrm{d} x(t)=y(t) \mathrm{d} t+g(t) \mathrm{d} \omega(t)
$$

Construct the following Lyapunov-Krasovskii functional described as

$$
V(x(t), t)=\sum_{i=1}^{4} V_{i}(x(t), t),
$$

where

$$
\begin{aligned}
& V_{1}(x(t), t)=x^{T}(t) P x(t), \\
& V_{2}(x(t), t)=\int_{-\bar{\tau}}^{0} \int_{t+\theta}^{t} y^{T}(s) R y(s) \mathrm{d} s \mathrm{~d} \theta,
\end{aligned}
$$

$$
\begin{aligned}
V_{3}(x(t), t)= & \int_{-\bar{\tau}}^{0} \int_{t+\theta}^{t} g^{T}(s) S g(s) \mathrm{d} s \mathrm{~d} \theta \\
V_{4}(x(t), t)= & \int_{t-\tau(t)}^{t} x^{T}(s) Q_{1} x(s) \mathrm{d} s \\
& +\int_{t-\bar{\tau}}^{t} x^{T}(s) Q_{2} x(s) \mathrm{d} s \\
& +\int_{t-\tau(t)}^{t} f^{T}(x(s)) Q_{3} f(x(s)) \mathrm{d} s .
\end{aligned}
$$

The weak infinitesimal operator $\mathscr{L}$ of the stochastic process $\{x(t), t \geqslant 0\}$ is given by

$$
\begin{aligned}
\mathscr{L} V_{1}= & 2 x^{T}(t) P y(t)+g^{T}(t) P g(t) \\
= & 2 x^{T}(t) P\left[-A x(t)+W_{0} f(x(t))\right. \\
& \left.+W_{1} f(x(t-\tau(t)))\right] \\
& +[C x(t)+D x(t-\tau(t))]^{T} \\
& \times P[C x(t)+D x(t-\tau(t))], \\
\mathscr{L} V_{2}= & \bar{\tau} y^{T}(t) R y(t)-\int_{t-\bar{\tau}}^{t} y^{T}(s) R y(s) \mathrm{d} s \\
= & \bar{\tau}\left[-A x(t)+W_{0} f(x(t))+W_{1} f(x(t-\tau(t)))\right]^{T} \\
& \times R\left[-A x(t)+W_{0} f(x(t))\right. \\
& \left.+W_{1} f(x(t-\tau(t)))\right]-\int_{t-\tau(t)}^{t} y^{T}(s) R y(s) \mathrm{d} s \\
& -\int_{t-\bar{\tau}}^{t-\tau(t)} y^{T}(s) R y(s) \mathrm{d} s, \\
\mathscr{L} V_{3}= & g^{T}(t) S g(t)-\int_{t-\bar{\tau}}^{t} g^{T}(s) S g(s) \mathrm{d} s \\
= & \bar{\tau}[C x(t)+D x(t-\tau(t))]^{T} \\
& \times S[C x(t)+D x(t-\tau(t))] \\
& -\int_{t-\tau(t)}^{t} g^{T}(s) S g(s) \mathrm{d} s \\
& -\int_{t-\bar{\tau}}^{t-\tau(t)} g^{T}(s) S g(s) \mathrm{d} s, \\
\mathscr{L} V_{4}= & x^{T}(t)\left(Q_{1}+Q_{2}\right) x(t)+f^{T}(x(t)) Q_{3} f(x(t)) \\
& -(1-\dot{\tau}(t)) x^{T}(t-\tau(t)) Q_{1} x(t-\tau(t)) \\
& -x^{T}(t-\bar{\tau}) Q_{2} x(t-\bar{\tau}) \\
& -(1-\dot{\tau}(t)) f^{T}(x(t-\tau(t))) Q_{3} f(x(t-\tau(t))) \\
\leqslant & x^{T}(t)\left(Q_{1}+Q_{2}\right) x(t)+f^{T}(x(t)) Q_{3} f(x(t)) \\
& -(1-\mu) x^{T}(t-\tau(t)) Q_{1} x(t-\tau(t)) \\
& (16) \\
&
\end{aligned}
$$




$$
\begin{aligned}
& -x^{T}(t-\bar{\tau}) Q_{2} x(t-\bar{\tau})-(1-\mu) \\
& \times f^{T}(x(t-\tau(t))) Q_{3} f(x(t-\tau(t))) .
\end{aligned}
$$

By Assumption 2.1, it is well known that there exist positive constants $\alpha>0$ and $\beta>0$ such that the following inequalities hold

$$
\begin{aligned}
& \alpha\left[x^{T}(t) G^{T} G x(t)-f^{T}(x(t)) f^{T}(x(t))\right] \geqslant 0, \\
& \beta\left[x^{T}(t-\tau(t)) G^{T} G x(t-\tau(t))\right. \\
& \left.-f^{T}(x(t-\tau(t))) f(x(t-\tau(t)))\right] \geqslant 0 .
\end{aligned}
$$

From (12), it follows that $x(t)-x(t-$ $\tau(t))-\int_{t-\tau(t)}^{t} y(s) \mathrm{d} s-\int_{t-\tau(t)}^{t} g(s) \mathrm{d} w(s)=0$ and $x(t-\tau(t))-x(t-\bar{\tau})-\int_{t-\bar{\tau}}^{t-\tau(t)} y(s) \mathrm{d} s-$ $\int_{t-\bar{\tau}}^{t-\tau(t)} g(s) \mathrm{d} w(s)=0$, then for any matrices $M=\left[\begin{array}{lllll}M_{1}^{T} & M_{2}^{T} & M_{3}^{T} & M_{4}^{T} & M_{5}^{T}\end{array}\right]$ and $N=$ $\left[\begin{array}{lllll}N_{1}^{T} & N_{2}^{T} & N_{3}^{T} & N_{4}^{T} & N_{5}^{T}\end{array}\right]$, we can obtain

$$
\begin{aligned}
& 2 \xi^{T}(t) M^{T}\left[x(t)-x(t-\tau(t))-\int_{t-\tau(t)}^{t} y(s) \mathrm{d} s\right. \\
& \left.-\int_{t-\tau(t)}^{t} g(s) \mathrm{d} w(s)\right]=0 \\
& 2 \xi^{T}(t) N^{T}\left[x(t-\tau(t))-x(t-\bar{\tau})-\int_{t-\bar{\tau}}^{t-\tau(t)} y(s) \mathrm{d} s\right. \\
& \left.-\int_{t-\bar{\tau}}^{t-\tau(t)} g(s) \mathrm{d} w(s)\right]=0
\end{aligned}
$$

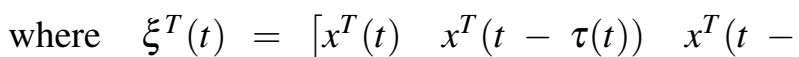
$\bar{\tau}) f^{T}(x(t)) f^{T}(x(t-\tau(t))]$. By Lemma 2.4, we have

$$
\begin{aligned}
- & 2 \xi^{T}(t) M^{T} \int_{t-\tau(t)}^{t} y(s) \mathrm{d} s \\
\leqslant & \tau(t) \xi^{T}(t) M^{T} R^{-1} M \xi(t) \\
& +\int_{t-\tau(t)}^{t} y^{T}(s) R y(s) \mathrm{d} s, \\
- & 2 \xi^{T}(t) N^{T} \int_{t-\bar{\tau}}^{t-\tau(t)} y(s) \mathrm{d} s \\
\leqslant & (\bar{\tau}-\tau(t)) \xi^{T}(t) N^{T} R^{-1} N \xi(t) \\
& +\int_{t-\bar{\tau}}^{t-\tau(t)} y^{T}(s) R y(s) \mathrm{d} s,
\end{aligned}
$$

$$
\begin{aligned}
- & 2 \xi^{T}(t) M^{T} \int_{t-\tau(t)}^{t} g(s) \mathrm{d} w(s) \\
\leqslant & \xi^{T}(t) M^{T} S^{-1} M \xi(t) \\
& +\left(\int_{t-\tau(t)}^{t} g(s) \mathrm{d} w(s)\right)^{T} S \int_{t-\tau(t)}^{t} g(s) \mathrm{d} w(s), \\
- & 2 \xi^{T}(t) N^{T} \int_{t-\bar{\tau}}^{t-\tau(t)} g(s) \mathrm{d} w(s) \\
\leqslant & \xi^{T}(t) N^{T} S^{-1} N \xi(t) \\
& +\left(\int_{t-\bar{\tau}}^{t-\tau(t)} g(s) \mathrm{d} w(s)\right)^{T} S \int_{t-\bar{\tau}}^{t-\tau(t)} g(s) \mathrm{d} w(s) .
\end{aligned}
$$

By considering (14)-(25), then we can eventually obtain

$$
\begin{aligned}
\mathscr{L} V \leqslant & \mathscr{L}_{1}(t)+\mathscr{L} V_{2}(t)+\mathscr{L} V_{3}(t)+\mathscr{L}_{4}(t) \\
& +\alpha\left[x^{T}(t) G^{T} G x(t)-f^{T}(x(t)) f^{T}(x(t))\right] \\
& +\beta\left[x^{T}(t-\tau(t)) G^{T} G x(t-\tau(t))\right. \\
& \left.-f^{T}(x(t-\tau(t))) f(x(t-\tau(t)))\right] \\
& +2 \xi^{T}(t) M^{T}[x(t)-x(t-\tau(t)) \\
& \left.-\int_{t-\tau(t)}^{t} y(s) \mathrm{d} s-\int_{t-\tau(t)}^{t} g(s) \mathrm{d} w(s)\right] \\
& +2 \xi^{T}(t) N^{T}[x(t-\tau(t))-x(t-\bar{\tau}) \\
& \left.-\int_{t-\bar{\tau}}^{t-\tau(t)} y(s) \mathrm{d} s-\int_{t-\bar{\tau}}^{t-\tau(t)} g(s) \mathrm{d} w(s)\right] \\
\leqslant & \xi^{T}(t)\left[\Xi+\tau(t) M^{T} R^{-1} M\right. \\
& \left.+(\bar{\tau}-\tau(t)) N^{T} R^{-1} N\right] \xi(t) \\
& -\int_{t-\tau(t)}^{t} g^{T}(s) S g(s) \mathrm{d} s \\
& -\int_{t-\bar{\tau}}^{t-\tau(t)} g^{T}(s) S g(s) \mathrm{d} s \\
& +\left(\int_{t-\tau(t)}^{t} g(s) \mathrm{d} w(s)\right)^{T} S \int_{t-\tau(t)}^{t} g(s) \mathrm{d} w(s) \\
& +\left(\int_{t-\bar{\tau}}^{t-\tau(t)} g(s) \mathrm{d} w(s)\right)^{T} S \int_{t-\bar{\tau}}^{t-\tau(t)} g(s) \mathrm{d} w(s), \\
= & \rho_{1}(t) \xi^{T}(t)\left[\Xi+\bar{\tau} M^{T} R^{-1} M\right] \xi(t) \\
& +\rho_{2}(t) \xi^{T}(t)\left[\Xi+\bar{\tau} N^{T} R^{-1} N\right] \xi(t) \\
&
\end{aligned}
$$




$$
\begin{aligned}
& -\int_{t-\tau(t)}^{t} g^{T}(s) S g(s) \mathrm{d} s-\int_{t-\bar{\tau}}^{t-\tau(t)} g^{T}(s) S g(s) \mathrm{d} s \\
& +\left(\int_{t-\tau(t)}^{t} g(s) \mathrm{d} w(s)\right)^{T} S \int_{t-\tau(t)}^{t} g(s) \mathrm{d} w(s) \\
& +\left(\int_{t-\bar{\tau}}^{t-\tau(t)} g(s) \mathrm{d} w(s)\right)^{T} S \int_{t-\bar{\tau}}^{t-\tau(t)} g(s) \mathrm{d} w(s),
\end{aligned}
$$

where

$$
\begin{aligned}
& \Xi=\Omega+\bar{\tau} \Xi_{1}^{T} R \Xi_{1}+\Xi_{2}^{T}(P+\bar{\tau} S) \Xi_{2} \\
& +M^{T} S^{-1} M+N^{T} S^{-1} N, \\
& \rho_{1}(t)=\tau(t) / \bar{\tau}, \rho_{2}(t)=(\bar{\tau}-\tau(t)) / \bar{\tau}, \\
& \xi(t)=\left[\begin{array}{lll}
x^{T}(t) & x^{T}(t-\tau(t)) & x^{T}(t-\bar{\tau}) f^{T}(x(t))
\end{array}\right. \\
& f^{T}(x(t-\tau(t))]^{T},
\end{aligned}
$$

and $\Omega, M, N, \Xi_{1}, \Xi_{2}$ are defined in Theorem 3.1. Since

$$
\begin{aligned}
& \mathrm{E}\left\{\int_{t-\tau(t)}^{t} g^{T}(s) S g(s) \mathrm{d} s\right\} \\
& =\mathrm{E}\left\{\left(\int_{t-\tau(t)}^{t} g(s) \mathrm{d} w(s)\right)^{T} S \int_{t-\tau(t)}^{t} g(s) \mathrm{d} w(s)\right\} \\
& \mathrm{E}\left\{\int_{t-\bar{\tau}}^{t-\tau(t)} g^{T}(s) S g(s) \mathrm{d} s\right\} \\
& =\mathrm{E}\left\{\left(\int_{t-\bar{\tau}}^{t-\tau(t)} g(s) \mathrm{d} w(s)\right)^{T} S \int_{t-\bar{\tau}}^{t-\tau(t)} g(s) \mathrm{d} w(s)\right\},
\end{aligned}
$$

Thus, if the matrix inequalities $\Xi+\bar{\tau} M^{T} R^{-1} M<0$, and $\Xi+\bar{\tau} N^{T} R^{-1} N<0$, then there exists a scalar $\lambda>0$ such that

$$
\mathrm{E}[\mathscr{L} V(x(t), t)] \leqslant-\lambda \mathrm{E}|x(t)|^{2},
$$

which implies that system (9) is asymptotically stable. Using Schur complement, (10) and (11) are equivalent to $\Xi+\bar{\tau} M^{T} R^{-1} M<0$ and $\Xi+$ $\bar{\tau} N^{T} R^{-1} N<0$, respectively. This completes the proof.

Remark 3.2 In Ref.23, the terms $-\int_{t-\bar{\tau}}^{t} y^{T}(s) R y(s) \mathrm{d} s$ and $-\int_{t-\bar{\tau}}^{t} g^{T}(s) S g(s) \mathrm{d} s$ were simply enlarged as $-\int_{t-\tau(t)}^{t} y^{T}(s) R y(s) \mathrm{d} s$ and $-\int_{t-\tau(t)}^{t} g^{T}(s) S g(s) \mathrm{d} s$ respectively. In this paper, $-\int_{t-\bar{\tau}}^{t-\tau(t)} y^{T}(s) R y(s) \mathrm{d} s$ and $-\int_{t-\bar{\tau}}^{t-\tau(t)} g^{T}(s) S g(s) \mathrm{d} s$ are reserved and the information on them are used.

Remark 3.3 In order to obtain the less conservative result, the sufficient condition for guaranteeing $\Xi+\tau(t) M^{T} R^{-1} M+(\bar{\tau}-\tau(t)) N^{T} R^{-1} N<0$ is not simply given by $\Xi+\bar{\tau} M^{T} R^{-1} M+\bar{\tau} N^{T} R^{-1} N<0$, but handled by two less conservativeness matrix inequalities $\Xi+\bar{\tau} M^{T} R^{-1} M<0$, and $\Xi+\bar{\tau} N^{T} R^{-1} N<$ 0 . The proposed method is so effective to reduce the conservativeness which will be illustrated by numerical examples.

Now, we will provide the robust stability criterion for uncertain neural networks (5) with the uncertainties satisfying (6).

Theorem 3.4 For given constants $\bar{\tau} \geqslant 0, \mu \geqslant 0$. Under Assumption 2.1, the system (5) is globally robustly asymptotically stable in the mean square, if there exist matrices $P>0, Q_{1}>0, Q_{2}>0, Q_{3}>$ $0, R>0, S>0, M_{i}, N_{i},(i=1,2, \cdots, 5)$, and scalars $\alpha>0, \beta>0, \varepsilon_{1}>0, \varepsilon_{2}>0, \varepsilon_{3}>0, \varepsilon_{4}>0$, such that the following LMIs hold

$$
\begin{aligned}
& {\left[\begin{array}{ccccc}
\Gamma_{1} & \bar{H}_{1} & \bar{H}_{2} & \varepsilon_{1} \bar{E}_{1}^{T} & \varepsilon_{2} \bar{E}_{2}^{T} \\
* & -\varepsilon_{1} I & 0 & 0 & 0 \\
* & * & -\varepsilon_{2} I & 0 & 0 \\
* & * & * & -\varepsilon_{1} I & 0 \\
* & * & * & * & -\varepsilon_{2} I
\end{array}\right]<0,} \\
& {\left[\begin{array}{ccccc}
\Gamma_{2} & \bar{H}_{1} & \bar{H}_{2} & \varepsilon_{3} \bar{E}_{1}^{T} & \varepsilon_{4} \bar{E}_{2}^{T} \\
* & -\varepsilon_{3} I & 0 & 0 & 0 \\
* & * & -\varepsilon_{4} I & 0 & 0 \\
* & * & * & -\varepsilon_{3} I & 0 \\
* & * & * & * & -\varepsilon_{4} I
\end{array}\right]<0,}
\end{aligned}
$$

where $\Gamma_{1}, \Gamma_{2}$ are defined in (10), (11), and

$$
\begin{aligned}
& \bar{H}_{1}=\left[\begin{array}{llllllllllll}
H^{T} P & 0 & 0 & 0 & 0 & \bar{\tau} H^{T} R & 0 & 0 & 0 & 0 & 0
\end{array}\right]^{T}, \\
& \bar{H}_{1}=\left[\begin{array}{lllllllllll}
0 & 0 & 0 & 0 & 0 & 0 & H^{T} P & \bar{\tau} H^{T} S & 0 & 0 & 0
\end{array}\right]^{T}, \\
& \bar{E}_{1}=\left[\begin{array}{lllllllllll}
-E_{1} & 0 & 0 & E_{2} & E_{3} & 0 & 0 & 0 & 0 & 0 & 0
\end{array}\right], \\
& \bar{E}_{2}=\left[\begin{array}{lllllllllll}
E_{4} & E_{5} & 0 & 0 & 0 & 0 & 0 & 0 & 0 & 0 & 0
\end{array}\right] .
\end{aligned}
$$

Proof. If we replace $A, W_{0}, W_{1}, C, D$ with $A+$ $H F(t) E_{1}, W_{0}+H F(t) E_{2}, W_{1}+H F(t) E_{3}, C+$ 
$H F(t) E_{4}$, and $D+H F(t) E_{5}$, respectively, then LMIs (10) and (11) are transformed into

$$
\begin{aligned}
& \Gamma_{1}+\bar{H}_{1} F(t) \bar{E}_{1}+\bar{E}_{1}^{T} F^{T}(t) \bar{H}_{1}^{T}+\bar{H}_{2} F(t) \bar{E}_{2} \\
& +\bar{E}_{2}^{T} F^{T}(t) \bar{H}_{2}^{T}<0, \\
& \Gamma_{2}+\bar{H}_{1} F(t) \bar{E}_{1}+\bar{E}_{1}^{T} F^{T}(t) \bar{H}_{1}^{T}+\bar{H}_{2} F(t) \bar{E}_{2} \\
& +\bar{E}_{2}^{T} F^{T}(t) \bar{H}_{2}^{T}<0 .
\end{aligned}
$$

According to the proof of Theorem 3.1, if inequalities (32),(33) hold, it is obvious that the uncertain stochastic neural networks (1) is globally robustly asymptotically stable in the mean square. By Lemma 2.5, for any scalars $\varepsilon_{1}>0, \varepsilon_{2}>0, \varepsilon_{3}>$ $0, \varepsilon_{4}>0$, we have

$$
\begin{aligned}
\Gamma_{1} & +\bar{H}_{1} F(t) \bar{E}_{1}+\bar{E}_{1}^{T} F^{T}(t) \bar{H}_{1}^{T}+\bar{H}_{2} F(t) \bar{E}_{2} \\
& +\bar{E}_{2}^{T} F^{T}(t) \bar{H}_{2}^{T} \\
\leqslant & \Gamma_{1}+\varepsilon_{1}^{-1} \bar{H}_{1} \bar{H}_{1}^{T}+\varepsilon_{1} \bar{E}_{1}^{T} \bar{E}_{1}+\varepsilon_{2}^{-1} \bar{H}_{2} \bar{H}_{2}^{T} \\
& +\varepsilon_{2} \bar{E}_{2}^{T} \bar{E}_{2}, \\
\Gamma_{2} & +\bar{H}_{1} F(t) \bar{E}_{1}+\bar{E}_{1}^{T} F^{T}(t) \bar{H}_{1}^{T}+\bar{H}_{2} F(t) \bar{E}_{2} \\
& +\bar{E}_{2}^{T} F^{T}(t) \bar{H}_{2}^{T} \\
\leqslant & \Gamma_{2}+\varepsilon_{3}^{-1} \bar{H}_{1} \bar{H}_{1}^{T}+\varepsilon_{3} \bar{E}_{1}^{T} \bar{E}_{1}+\varepsilon_{4}^{-1} \bar{H}_{2} \bar{H}_{2}^{T} \\
& +\varepsilon_{4} \bar{E}_{2}^{T} \bar{E}_{2} .
\end{aligned}
$$

Therefore, if $\quad \Gamma_{1}+\varepsilon_{1}^{-1} \bar{H}_{1} \bar{H}_{1}^{T}+\varepsilon_{1} \bar{E}_{1}^{T} \bar{E}_{1}+$ $\varepsilon_{2}^{-1} \bar{H}_{2} \bar{H}_{2}^{T}+\varepsilon_{2} \bar{E}_{2}^{T} \bar{E}_{2}<0$, and $\Gamma_{2}+\varepsilon_{3}^{-1} \bar{H}_{1} \bar{H}_{1}^{T}+$ $\varepsilon_{3} \bar{E}_{1}^{T} \bar{E}_{1}+\varepsilon_{4}^{-1} \bar{H}_{2} \bar{H}_{2}^{T}+\varepsilon_{4} \bar{E}_{2}^{T} \bar{E}_{2}<0$, then (32) and (33) hold. By Schur complement, (30) and (31) are equivalent to $\Gamma_{1}+\varepsilon_{1}^{-1} \bar{H}_{1} \bar{H}_{1}^{T}+\varepsilon_{1} \bar{E}_{1}^{T} \bar{E}_{1}+$ $\varepsilon_{2}^{-1} \bar{H}_{2} \bar{H}_{2}^{T}+\varepsilon_{2} \bar{E}_{2}^{T} \bar{E}_{2}<0$, and $\Gamma_{2}+\varepsilon_{3}^{-1} \bar{H}_{1} \bar{H}_{1}^{T}+$ $\varepsilon_{3} \bar{E}_{1}^{T} \bar{E}_{1}+\varepsilon_{4}^{-1} \bar{H}_{2} \bar{H}_{2}^{T}+\varepsilon_{4} \bar{E}_{2}^{T} \bar{E}_{2}<0$, respectively. This completes the proof.

As a especial case, we consider the robust stability of the following neural network

$$
\begin{aligned}
\dot{x}(t)= & -(A+\Delta A) x(t)+\left(W_{0}+\Delta W_{0}\right) f(x(t)) \\
& +\left(W_{1}+\Delta W_{1}\right) f(x(t-\tau(t))),
\end{aligned}
$$

where the activation function $f($.$) satisfies Assump-$ tion 2.2.
Theorem 3.5 For given constants $\bar{\tau} \geqslant 0, \mu \geqslant 0$, and diagonal matrix $K=\operatorname{diag}\left\{k_{1}, k_{2}, \cdots, k_{n}\right\}$. Under Assumption 2.2, the system (36) is globally robustly asymptotically stable, if there exist matrices $P>0, Q_{1}>0, Q_{2}>0, Q_{3}>0, R>0, M_{i}, N_{i},(i=$ $1,2, \cdots, 5)$, diagonal matrices $D_{1} \geqslant 0, D_{2} \geqslant 0$, and scalars $\alpha>0, \beta>0, \varepsilon_{1}>0, \varepsilon_{2}>0$, such that the following LMIs hold

$$
\begin{aligned}
& {\left[\begin{array}{cccc}
\Sigma & \bar{\tau} \widetilde{M}^{T} & \widetilde{H} & \varepsilon_{1} \widetilde{E}^{T} \\
* & -\bar{\tau} R & 0 & 0 \\
* & * & -\varepsilon_{1} I & 0 \\
* & * & * & -\varepsilon_{1} I
\end{array}\right]<0,} \\
& {\left[\begin{array}{cccc}
\Sigma & \bar{\tau} \widetilde{N}^{T} & \widetilde{H} & \varepsilon_{1} \widetilde{E}^{T} \\
* & -\bar{\tau} R & 0 & 0 \\
* & * & -\varepsilon_{2} I & 0 \\
* & * & * & -\varepsilon_{2} I
\end{array}\right]<0,}
\end{aligned}
$$

where

$$
\begin{aligned}
\Sigma & =\left[\begin{array}{cccccc}
\Sigma_{11} & \Sigma_{12} & \Sigma_{13} & \Sigma_{14} & \Sigma_{15} & -\bar{\tau} A^{T} R \\
* & \Sigma_{22} & \Sigma_{23} & \Sigma_{24} & \Sigma_{25} & 0 \\
* & * & \Sigma_{33} & -N_{4}^{T} & -N_{5}^{T} & 0 \\
* & * & * & \Sigma_{44} & \Sigma_{45} & \bar{\tau} W_{0}^{T} R \\
* & * & * & * & \Sigma_{55} & \bar{\tau} W_{1}^{T} R \\
* & * & * & * & * & -\bar{\tau} R
\end{array}\right], \\
\widetilde{M} & =\left[\begin{array}{llllll}
M_{1}^{T} & M_{2}^{T} & M_{3}^{T} & M_{4}^{T} & M_{5}^{T} & 0
\end{array}\right], \\
\widetilde{N} & =\left[\begin{array}{llllll}
N_{1}^{T} & N_{2}^{T} & N_{3}^{T} & N_{4}^{T} & N_{5}^{T} & 0
\end{array}\right], \\
\widetilde{H} & =\left[\begin{array}{llllll}
H^{T} P & 0 & 0 & 0 & 0 & \bar{\tau} H^{T} R
\end{array}\right]^{T}, \\
\widetilde{E} & =\left[\begin{array}{llllll}
-E_{1} & 0 & 0 & E_{2} & E_{3} & 0
\end{array}\right],
\end{aligned}
$$

and

$$
\begin{aligned}
& \Sigma_{11}=-P A-A^{T} P^{T}+Q_{1}+Q_{2}+M_{1}+M_{1}^{T}, \\
& \Sigma_{12}=-M_{1}+M_{2}^{T}+N_{1}, \\
& \Sigma_{13}=M_{3}^{T}-N_{1}, \\
& \Sigma_{14}=P W_{0}+M_{4}^{T}+K L_{1}, \\
& \Sigma_{15}=P W_{1}+M_{5}^{T}, \\
& \Sigma_{22}=-(1-\mu) Q_{1}-M_{2}-M_{2}^{T}+N_{2}+N_{2}^{T}, \\
& \Sigma_{23}=-M_{3}^{T}-N_{2}+N_{3}^{T}, \\
& \Sigma_{24}=-M_{4}^{T}+N_{4}^{T},
\end{aligned}
$$




$$
\begin{aligned}
& \Sigma_{25}=-M_{5}^{T}+N_{5}^{T}+K L_{2}, \\
& \Sigma_{33}=-N_{3}-N_{3}^{T}-Q_{2}, \\
& \Sigma_{44}=-2 L_{1}+Q_{3}, \\
& \Sigma_{55}=-2 L_{2}-(1-\mu) Q_{3} .
\end{aligned}
$$

Proof. Choosing Lyapunov-Krasovskii functional $V(x(t), t)=V_{1}(x(t), t)+V_{2}(x(t), t)+V_{4}(x(t), t)$, where $V_{1}(x(t), t), V_{2}(x(t), t)$, and $V_{4}(x(t), t)$ are defined in (13). By Assumption 2.2, it is well known that there exist diagonally matrices $D_{1} \geqslant 0, D_{2} \geqslant 0$ such that the following inequalities hold

$$
\begin{aligned}
& 2\left[x^{T}(t) K L_{1} f(x(t))-f^{T}(x(t)) L_{1} f(x(t))\right] \geqslant 0, \\
& 2\left[x^{T}(t-\tau(t)) K L_{2} f(x(t-\tau(t)))\right. \\
& \left.\quad-f^{T}(x(t-\tau(t))) L_{2} f(x(t-\tau(t)))\right] \geqslant 0 .
\end{aligned}
$$

According to the proof of Theorems 3.1 and 3.4, the Theorem 3.5 can be easily obtained.

Remark 3.6 When time-varying delay $\tau(t)$ is not differentiable, the stability criteria can be easily obtained by setting $Q_{1}=0, Q_{3}=0$ in Theorems 3.1 and 3.4-3.5.

\section{Numerical Examples}

Example 4.1 Consider the neural networks (36) with time-varying delay and the following parameters $^{12}$

$$
\begin{gathered}
A=\left[\begin{array}{ll}
2 & 0 \\
0 & 2
\end{array}\right], W_{0}=\left[\begin{array}{cc}
1 & 1 \\
-1 & -1
\end{array}\right], W_{1}=\left[\begin{array}{cc}
0.88 & 1 \\
1 & 1
\end{array}\right] \\
\Delta A=0, \Delta W_{0}=0, \Delta W_{1}=0, k_{1}=0.4, k_{2}=0.8
\end{gathered}
$$

For this example, the corresponding maximum allowable delay bounds $\bar{\tau}$ for various $\mu$ can be obtained by using Theorem 3 . For a detailed comparison with the results in Refs.12-14, we made Table 1. According to Table 1, it is seen that Theorem 3.5 improves some existing results ${ }^{12-14}$.

Table 1. delay bounds for different $\mu$.

\begin{tabular}{cccc}
\hline$\mu$ & 0.8 & 0.9 & unknown \\
\hline Refs.12 and 13 & 1.2281 & 0.8636 & 0.8298 \\
Ref.14 & 1.6831 & 1.1493 & 1.0880 \\
Theorem 3.5 & 2.3534 & 1.6050 & 1.5103 \\
\hline
\end{tabular}

Example 4.2 Consider the stochastic neural networks (1) with the following parameters ${ }^{24}$

$$
\begin{aligned}
& A=\left[\begin{array}{ll}
4 & 0 \\
0 & 5
\end{array}\right], W_{0}=\left[\begin{array}{cc}
0.4 & -0.7 \\
0.1 & 0
\end{array}\right], \\
& W_{1}=\left[\begin{array}{cc}
-0.2 & 0.6 \\
0.5 & -0.1
\end{array}\right], C=\left[\begin{array}{cc}
0.5 & 0 \\
0 & 0.5
\end{array}\right], \\
& D=\left[\begin{array}{cc}
0.5 & -0.5 \\
-0.5 & 0
\end{array}\right], G=0.5 I, \\
& H=\left[\begin{array}{ll}
0.1 & -0.1
\end{array}\right]^{T}, E_{1}=\left[\begin{array}{ll}
0.2 & 0.3
\end{array}\right], \\
& E_{2}=\left[\begin{array}{ll}
0.2 & -0.3
\end{array}\right], E_{3}=\left[\begin{array}{ll}
-0.2 & -0.3
\end{array}\right], \\
& E_{4}=\left[\begin{array}{ll}
0 & 0
\end{array}\right], E_{5}=\left[\begin{array}{ll}
0 & 0
\end{array}\right],
\end{aligned}
$$

and time-varying delay satisfying $0 \leqslant \tau(t) \leqslant \bar{\tau}$.

For this example, the results in Refs.18-19 can not conclude whether this system is asymptotically or not. Applying the result in Ref.23 to this system, the achieved maximum allowable delay bound is $\bar{\tau}=0.4109$. However, using Theorem 3.4 in this paper with $Q_{1}=Q_{3}=0$, we can obtain the larger allowable delay bound $\bar{\tau}=0.8255$. For this example, it is obvious that the results in this paper are less conservative than those in Refs.18,19 and 23.

\section{Conclusion}

In this paper, the robust stability problem is investigated for a class of uncertain stochastic neural networks with time-varying delay and parameter uncertainties. By using the Lyapunov functional method and by resorting to the novel enlargement method for estimating the upper bound of the stochastic derivative of Lyapunov functional, we obtain the less conservative stability criteria in terms of linear matrix inequalities (LMIs). Finally, two numerical examples are given to show the effectiveness and benefits of the proposed method.

\section{Acknowledgments}

This work was supported by National Natural Science Foundation of China (No. 60850004).

1. J. J. Hopfileld, "Neurons with graded response have collective computational properties like those of twosate neurons," Proc. Natl. Acad. Sci., 81, 3088-3092 (1984). 
2. C. M. Marcus, R. M. Westervelt, "Stability of analog neural networks with delay," Phys. Rev. A, 39, 347359 (1989).

3. S. Arik and V. Tavsanoglu, "Equilibrium analysis of delayed CNNs," IEEE Trans. Circuits Syst. I, Fundam. Theory Appl., 45, 168-171 (1998).

4. J. Cao," "Global stability conditions for delayed CNNs," IEEE Trans. Circuits Syst. I, Fundam. Theory Appl., 48, 1330-1333 (2001).

5. X. Liao, G. Chen, and E. N. Sanchez, "LMI-based approach for asymptotically stability analysis of delayed neural networks," IEEE Trans. Circuits Syst. I, Fundam. Theory Appl., 49, 1033-1039 (2002).

6. S. Arik, "Global asymptotic stability of a larger class of delayed cellular neural networks with constant delay," Phys. Lett. A, 311, 504-511 (2003).

7. V. Singh, "Simplified LMI condition for global asymptotic stability of delayed neural networks," Chaos, Solitons \& Fractals, 29, 470-473 (2006).

8. S. Xu, Y. Chu, J. Lu, "New results on global exponential stability of recurrent neural networks with timevarying delays," Phys. Lett. A, 352, 371-379 (2006).

9. T. Ensari, S. Arik, "Global stability of class of neural networks with time varying delays," IEEE Trans. Circuits Syst. II, Exp. Briefs, 52, 126-130 (2005).

10. S. Xu, J. Lam, D. W. C. Ho, Y. Zou, ”Novel global asymptotic stability criteria for delayed cellular neural networks," IEEE Trans. Circuits Syst. -II, Exp. Briefs, 52, 349-353 (2005).

11. H. J. Cho, J. H. Park, "Novel delay-dependent robust stability criterion of delayed cellular neural networks," Chaos, Solitons \& Fractals, 32, 1194-1200 (2007).

12. H. L. Liu, G. H. Chen, "Delay-dependent stability for neural networks with time-varying delay," Chaos, Solitons \& Fractals, 33, 171-177 (2007).

13. C. C. Hua, C. N. Long, X. P. Guan, "New results on stability analysis of neural networks with timevarying delays," Phys. Lett. A, 352, 335-340 (2006).

14. Y. He, G. P. Liu, D. Rees, "New delay-dependent stability criteria for neural networks with time-varying delays," IEEE Trans. Neural Netw., 18, 310-314 (2007).
15. S. Blythe, X. Mao, and X. Liao, "Stability of stochastic delay neural networks," J. Franklin Inst., 338, 481495 (2001).

16. L. Wan, J. Sun, "Mean square exponential stability of stochastic delayed Hopfield neural networks Phys. Lett. A, 343, 306-318 (2005).

17. Y. Liu, Z. Wang, X. Liu, "On global exponential stability of generalized stochastic neural networks with mixed time-delays," Neurocomputing, 70, 314-326 (2006).

18. Z. Wang, H. Shu, J. Fang and X. Liu, "Robust stability for stochastic Hopfield neural networks with time delays," Nonlinear Anal.: Real World Appl., 7, 11191128 (2006).

19. J. Zhang, P. Shi, J. Qiu, "Novel robust stability criteria for uncertain stochastic Hopfield neural networks with time-varying delays," Nonlinear Anal.: Real World Appl., 8, 1349-1357 (2007).

20. H. Huang, J. Cao, "Exponential stability analysis of uncertain stochastic neural networks with multiple delays," Nonlinear Anal.: Real World Appl., 8, 646-653 (2007).

21. Z. Wang, S. Lauria, J. Fang and X. Liu, "Exponential stability of uncertain stochastic neural networks with mixed time-delays," Chaos, Solitons \&Fractals, 32, 62-72 (2007).

22. H. Zhao, N. Ding, "Dynamic analysis of stochastic bidirectional associative memory neural networks with delays," Chaos, Solitons \&Fractals, 32, 1692 1702 (2007).

23. H. Huang, G. Feng, "Delay-dependent stability for uncertain stochastic neural networks with time-varying delay," Physica A: Stat. Mech. Appl., 381, 93-103 (2007).

24. H. Huang, G. Feng, "Corrigendum to "Delaydependent stability for uncertain stochastic neural networks with time-varying delay" [Physica A 381 (2007) 93-103],"Physica A: Stat. Mech. Appl., 387, 1431-1432 (2008).

25. X. Mao, Stochastic Differential Equations and Their Applications, U. K.:Horwood (1997). 